\title{
Impact of different physical activities on executive functioning
}

\author{
K. Sneidere ${ }^{1}$, J. Harlamova ${ }^{1}$, V. Arnis ${ }^{1}$, Z. Ulmane ${ }^{1}$, I. Mintale ${ }^{2}$, K. Kupcs ${ }^{2}$, and A. Stepens ${ }^{1}$ \\ ${ }^{1}$ Rīga Stradiņš University, Riga, Latvia \\ ${ }^{2}$ Pauls Stradins Clinical University Hospital, Riga, Latvia
}

\begin{abstract}
The aging of the Western society has become one of the key issues in research. Lately research has shown relationship between improvement in cognitive functioning and involvement in physical activities. The aim of the study was to examine the impact of different types of physical activities on executive functioning. Overall 30 seniors aged from 65 participated in a two-day study. They were divided into three groups per their life-style - aerobic activity, strength training and sedentary. Results indicated differences between the groups in cognitive efficiency $(F=6.791, p<0.01)$ and working memory $(F=6.179, p<0.01)$ as well as the inhibition of attention $(F=4.311, p<0.01)$. The mean scores were higher in the aerobic group. The results indicate that involvement in aerobic physical activity might lessen the decline in executive functioning in seniors.
\end{abstract}

\section{Introduction}

WHO prognosis is that by year 2050 population aged over 60 will almost double, which means that approx $22 \%$ of population will meet difficulties associated with aging, one of them being decline in cognitive functioning [1]. Research has shown that with aging there is a decrease in the brain volume, especially, in frontal, parietal and temporal cortices [2], which leads to decline in cognitive functioning, for example, there are indications of decline in executive functions [3,4].

Executive function is broad construct of attention thought to be involved in higher level cognitive tasks performed in the service of a goal [5]. Executive functions have been researched extensively and there are several theories on the division of the functions. One of the theories involves three core executive functions - inhibition - which involves being able to control your attention, behaviour, thoughts and emotions to override a dominant reaction, for example, inhibitory control of attention allows us to focus on our task at hand. Working memory is another core function and involves ability to work with information mentally holding it in mind, for example, doing math. Cognitive flexibility refers to ability to adjust, change perspectives, it is involved in problem solving (for example, thinking outside the box) [6].

More and more research results indicate an increase in grey and white matter volume in prefrontal cortex as well as increased functioning of key nodes in the executive control network in people who are involved in aerobic exercises [7]. Evidence of greater brain volume in temporal brain regions in physical active older adults in comparison with less 
active older adults can be found as well [8]. Increasing amount of research suggests increase of volume in hippocampus and reduced brain atrophy due to physical exercise [7,9].

\section{The present study}

Aim of the present study is to examine the impact of different types of physical activities on executive functioning, focusing on inhibition of attention and working memory. We hypothesised that participants involved in aerobic physical exercise will show better results than participants involved in strength training with aerobic activity elements in lifestyle or participants with sedentary lifestyle. This study was funded/partly funded by the Latvian National Research Programme Biomedicine for Public Health (BIOMEDICINE) 2014-2017.

\section{Materials and methods}

\subsection{Participants}

To assess the impact of different types of physical activities on executive function, 30 participants aged $65-80(M=72.27, \mathrm{SD}=4.55)$ were included in the data analysis. $30 \%$ of the participants were male. Most of the participants had higher education $(53.3 \%)$, secondary/secondary professional education $(33.2 \%)$ or incomplete higher education $(10 \%)$. Only one participant had less than secondary education $(3.3 \%)$. The mean years spent in education were 15, longest being 22 years (see Table 1). All of the participants were native Latvian speakers, who use this language daily.

Table 1. Participant characteristics.

\begin{tabular}{lccc}
\hline \multicolumn{1}{c}{ Characteristic } & Mean & Range & SD \\
\hline Age & 72.27 & $65-80$ & 4.55 \\
Education (y) & 15 & $7-22$ & 3.69 \\
\hline
\end{tabular}

Note. SD-standard deviation.

Seniors were divided into three groups - seniors involved in regular aerobic activities (e.g. orienting, running, swimming, Nordic Walking, etc.), seniors involved in regular strength training (e.g. long jumping, hammer throw, etc.) with irregular aerobic activities, such as walking daily or running, and seniors with sedentary lifestyle. Both physically active groups included participants involved in physical activities for leisure and participants involved in physical activities as competitive athletes.

We indicated several inclusion/exclusion criteria regarding language and medical history that might impact the results of cognitive tests, such as known history of cardio-vascular diseases, metabolic disorders, pulmonary and respiratory diseases, ongoing oncologic diseases, rheumatologic diseases and mental diseases (for detailed description see Table 2).

\subsection{Instruments}

For overall screening, Montreal Cognitive Assessment Scale (MoCA) [10] was used. This test consists of twelve tasks, assessing visual-spatial perception, executive functions, short and long-term memory as well as working memory, attention, thinking processes, language skills and orientation in time and place. The maximal count of points is 30 , the accepted norm is 26 points. 
Table 2. Inclusion/exclusion criteria.

\begin{tabular}{ll}
\hline \multicolumn{1}{c}{$\begin{array}{c}\text { Inclusion/exclusion } \\
\text { criteria }\end{array}$} & \multicolumn{1}{c}{ Description } \\
\hline Language & native Latvian speaker \\
No history of: & $\begin{array}{l}\text { uncontrolled arterial hypertension }[>140 />80 \mathrm{mmHg}], \\
\text { angina pectoris, } \\
\text { cardiac failure, } \\
\text { stroke } \\
\text { diabetes, } \\
\text { obesity }[\mathrm{BMI}>30] \\
\text { require regular inhaler use }\end{array}$ \\
$\begin{array}{l}\text { metabolic disorders as } \\
\text { pulmonary and respiratory diseases as } \\
\text { ongoing oncologic disease } \\
\text { rheumatologic diseases that } \\
\text { mental diseases as }\end{array}$ & $\begin{array}{l}\text { require regular painkiller use } \\
\text { depression, } \\
\text { dementia, } \\
\text { ongoing schizophrenia that require medication }\end{array}$ \\
\hline
\end{tabular}

To assess working memory, The Numbers Reversed test [11] was used. In this test, participants are presented with a series of digits which must be repeated in reverse order. The norms for this test depend on the age of the participant.

To assess inhibition of attention 3 instruments was used:

- Visual Matching test [11]. This test requires crossing out similar digits from a sequence of digits with time limit.

- Map Test of Everyday Attention [12]. This task requires finding and circling an icon of a gas station on a city map within the time limit of two minutes. For the first minute, participant is asked to circle the icons with one colour (e.g. green), and for the second minute, participant is asked to circle the icons with different colour (e.g. red). There are a total of 80 gas station icons. The aim of this test is to measure the selective attention.

- Reaction time test [13]. This is a computer-based test, used to measure simple reaction time and the complex reaction time and consists of two tasks. In the first task, the simple reaction time was measured. The participants were presented with a picture of an athlete. In a randomized period of time, a black ball appeared by the athlete's left hand. The participants had to press a "STOP" button as soon as the ball appeared. The second task measured complex reaction time. In this task, participants were presented with the same picture of an athlete; however, this time the black ball would appear in either of two squares next to athlete's left hand. Depending from the square, participants had to react by pressing "E" or "U" on the keyboard.

To determine the physical activity of each participant, two questionnaires were used International Physical Activity Questionnaire for Elderly (IPAQ-E) [14] and a modified version of Social Determinants of Health Behaviours [15]. IPAQ-E questionnaire consists of four questions regarding the physical activity in the last seven days - time spent sitting, time spent walking, time spent doing moderate physical activities and time spent doing vigorous physical activities. The data were measured in minutes and multiplied by a fixed coefficient. To identify the overall tendencies in each of the participant's lifestyle, a modified version of Social Determinants of Health Behaviours questionnaire was used. 
This questionnaire includes questions regarding their physical activity over their course of life (e.g., what kind of aerobic exercises have you been involved during your life?), the regularity of the exercises and activities for the past years, months and weeks. The questionnaire also includes questions that reflect participants' habits of alcohol use, smoking, and diet.

\subsection{Procedure}

Data collection for each participant lasted for two days. On the first day, the assessment of executive functioning was conducted, on the second day the data on each participant's lifestyle was obtained. Ethical approval has been obtained from the Riga Stradiņš University Ethics Committee. Participation was voluntary and written consent was obtained from each of the participants prior to data collection.

\subsection{Data analysis}

SPSS version 21. (Statistical Package for the Social Sciences) was used to process the data. The data was analysed with Spearman correlation coefficient and ANOVA analysis with Post Hoc test (LSD).

\section{Results}

The mean results of the tests assessing executive functioning were calculated.

Table 3. Mean results of the cognitive assessment tests.

\begin{tabular}{lrccc}
\hline \multicolumn{1}{c}{ Test } & Group & Mean & SD & ND \\
\hline MoCA & & 25.3 & 2.48 & .065 \\
& Aerobic group & 26.08 & 2.18 & .114 \\
& Strength training group & 25 & 1.85 & .769 \\
The Map Search test & Sedentary group & 24.56 & 3.24 & .679 \\
(Minute 1) & & 30.13 & 11.22 & 1 \\
& & & & \\
& Aerobic group & 34.23 & 9.97 & .999 \\
& Strength training group & 31.75 & 13.89 & .907 \\
The Map Search Test & Sedentary group & 22.78 & 6.92 & .928 \\
(Minute 2) & & 23.73 & 7.63 & .653 \\
& & & & \\
& & 26.85 & 6.59 & .777 \\
The Map Search Test & Aerobic group & 21.75 & 7.44 & .873 \\
(total) & Strength training group & 21 & 8.32 & .879 \\
& Sedentary group & 21 & .076 \\
& & 53.87 & 15.06 & .076 \\
& & & & \\
Simple reaction time & Aerobic group & 61.08 & 9.31 & .192 \\
& Strength training group & 53.5 & 18.15 & .361 \\
& Sedentary group & 43.78 & 14.32 & .596 \\
& & .417 & .268 & .011 \\
& Aerobic group & .349 & .05 & .878 \\
& Strength training group & .345 & .09 & .369 \\
& Sedentary group & .580 & .455 & .209
\end{tabular}


Complex reaction time

Cognitive efficiency

The Numbers

Reversed test
.693

.280

.021

$\begin{array}{rccc}\text { Aerobic group } & .609 & .09 & .874 \\ \text { Strength training group } & .692 & .16 & .363 \\ \text { Sedentary group } & .816 & .471 & .424 \\ & 102.5 & 13.25 & .767 \\ \text { Aerobic group } & 110.85 & 9.45 & .990 \\ \text { Strength training group } & 98.75 & 15.68 & .387 \\ \text { Sedentary group } & 93.78 & 8.60 & .880 \\ & 101.47 & 12.71 & .859 \\ \text { Aerobic group } & 107 & 10.48 & .885 \\ \text { Strength training group } & 99 & 15.02 & .893 \\ \text { Sedentary group } & 95.67 & 11.38 & .931 \\ & 102.10 & 11.50 & .997\end{array}$

Aerobic group $\quad 109.23 \quad 8.97 \quad 938$

Strength training group $\quad 98.25 \quad 11.17 \quad .956$

Sedentary group $\quad 95.22 \quad 9.96 \quad .998$

Note: $S D=$ Standard Deviation, ND - Normal Distribution.

In all tests participants, involved in a life style with aerobic physical activities, showed higher results than participants with strength training or sedentary lifestyle. When compared with the means from the sedentary life style group, the group with strength training indicated higher results (see Table 3 ).

To determine whether there are significant differences across the three groups ANOVA with LSD correction was used. There were significant differences between the groups in cognitive efficiency $(F=6.791, p<0.01)$ and working memory $(F=6.179, p<0.01)$. Differences between groups were also found in the total score of the Map Search Test which was used to measure the inhibition of attention $(F=4.311, p<0.01)$ (see Table 4$)$.

Table 4. Differences across three groups of different physical activities.

\begin{tabular}{lll}
\hline \multicolumn{1}{c}{ Test } & $\boldsymbol{F}$ & Sig. \\
\hline MoCA & 1.105 & .346 \\
The Map Search Test & $\mathbf{4 . 3 1 1}$ & $\mathbf{0 2 4}$ \\
(total score) & & \\
Simple reaction time & 2.648 & .089 \\
Complex raction time & 1.484 & .245 \\
Cognitive efficiency & $\mathbf{6 . 7 9 1}$ & $\mathbf{. 0 0 4}$ \\
Visual Matching test & 2.572 & .095 \\
The Numbers Reversed & $\mathbf{6 . 1 7 9}$ & $\mathbf{. 0 0 8}$ \\
test & & \\
\hline
\end{tabular}

To examine the relationship between the physical activity and executive function, MET minutes for walking, moderate activity and vigorous activity were collated with the results from tests measuring executive function, Spearman correlation coefficient was used. There was a statistically significant correlation only between the complex reaction time and MET minutes for walking $\left(r_{\mathrm{s}}=-.396, p<0.05\right)$ and complex reaction time and MET minutes for vigorous physical activity $\left(r_{\mathrm{s}}=-.436, p<0.05\right)$. 


\section{Discussion}

The aim of this study was to examine the impact of different types of physical activities on executive functioning, focusing on inhibition of attention and working memory. The results supported our hypothesis that participants involved in aerobic physical exercise would show higher results than participants involved in strength training with aerobic activity elements in lifestyle, or participants with sedentary lifestyle. Participants involved in aerobic physical activities (such as walking, Nordic Walking, swimming, cycling etc.) did show higher results in cognitive testing, which complies with the evidence given by Enzo Iuliano [16] or Geoffroy Boucard [3].

As predicted, there were differences in working memory scores across all three groups. Working memory is a memory system that involves holding information and mentally manipulating it. It can be either verbal or visual-spatial and is essential in seeing connections, making sense of written or spoken language, doing maths, translating instructions into action plans, etc. [6], thus being one of the essential parts of executive functions. Working memory is associated with the grey matter volume in frontal-parietal circuit [17], which is one of the areas strongly impacted by ageing [2] and is also the area of brain, where an increase in the volume of grey and white brain matter is observed after an aerobic physical activity intervention $[7,8]$. This indicates that the higher results in the aerobic activity group were consequential rather than circumstantial.

The concept of cognitive efficiency here is defined as the capacity of the cognitive system to process information automatically. This process includes processing speed and short-term memory [11]. Though there has been research on the impact of cognitive training on cognitive efficiency (e.g. [18]. However, research on processing speed indicates a relationship between physical activity and improved processing speed. For example, research indicates that higher fitness improves processing speed in memory tasks [7].

This study has several limitations. Firstly, the sample of the participants is still very small and the results are not statistically relevant and might change with the increase in sample. Secondly, we did not check for education, which might impact the results. Thirdly, we did not check for the longevity of the physical activities, as well as for the previous experience, focusing mainly on the current physical activity status.

\section{Conclusions}

The topic of impact of different physical activities on the executive functions has yet to be researched more thoroughly. However, the results of this study indicate that there are differences across the groups in such executive functions as working memory and processing speed, allowing us to conclude that the involvement in aerobic physical activities could in fact lessen the decline in executive functioning in the aging population. Results also indicated relationship between walking and processing speed and processing speed and regular involvement in vigorous aerobic activities, which also indicates benefits not only from regular vigorous aerobic activities, but also from as simple activity as a daily walk.

This study was funded/partly funded by the Latvian National Research Programme Biomedicine for Public Health (BIOMEDICINE) 2014-2017.

\section{References}

1 World Health Organization, "World report on Ageing And Health," 2015

2 S.J. Colcombe, K.I. Erickson, N. Raz, A.G. Webb, N.J. Cohen, E. Mcauley, and A.F. Kramer, "Aerobic Fitness Reduces Brain Tissue Loss in Aging Humans," 58, 
no. 2, pp. 176-180, 2003

3 G.K. Boucard, C.T. Albinet, A. Bugaiska, C.A. Bouquet, D. Clarys, and M. Audiffren, "Impact of Physical Activity on Executive Functions in Aging: A Selective Effect on Inhibition Among Old Adults," pp. 808-827, 2012

4 M.W. Voss, S. Heo, R.S. Prakash, K.I. Erickson, H. Alves, L. Chaddock, A.N. Szabo, E.L. Mailey, S.M. White, N. Gothe, T.R. Wo, E. Mcauley, B.P. Sutton, and A.F. Kramer, "The Influence of Aerobic Fitness on Cerebral White Matter Integrity and Cognitive Function in Older Adults: Results of a One-Year Exercise Intervention," 2985, no. February 2012, pp. 2972-2985, 2013

5 L. Vaughan and K. Giovanello, "Executive function in daily life: Age-related influences of executive processes on instrumental activities of daily living.," Psychol. Aging, 25, no. 2, pp. 343-355, Jun. 2010

6 A. Diamond, "Executive Functions," Annu. Rev. Psychol., 64, no. 1, pp. 135-168, Jan. 2013

7 K.I. Erickson, M.W. Voss, R. Shaurya, C. Basak, and A. Szabo, "Exercise training increases size of hippocampus and improves memory," 108, no. 7, pp. 3017-3022, 2011

8 C. Benedict, S.J. Brooks, J. Kullberg, R. Nordenskjöld, J. Burgos, M. Le, L. Kilander, E. Larsson, L. Johansson, H. Ahlström, L. Lind, and H.B. Schiöth, "Association between physical activity and brain health in older adults," $N B A, \mathbf{3 4}$, no. 1, pp. 83-90, 2013

9 V.R. Varma, Y.-F. Chuang, G.C. Harris, E.J. Tan, and M.C. Carlson, "Lowintensity daily walking activity is associated with hippocampal volume in older adults.," Hippocampus, 25, no. 5, pp. 605-15, May 2015

10 Z.S. Nasreddine, N.A. Phillips, V. BÃ@dirian, S. Charbonneau, V. Whitehead, I. Collin, J.L. Cummings, and H. Chertkow, "The Montreal Cognitive Assessment, MoCA: A Brief Screening Tool For Mild Cognitive Impairment," J. Am. Geriatr. Soc., 53, no. 4, pp. 695-699, Apr. 2005

11 N. Woodcock, R.W. McGrew, K.S. Mather, Woodcock-Johnson III Test Manual, III. Itasca, IL: Riverside Publishing Company, 2001

12 I.A.N.H. Robertson, T. Ward, V. Ridgeway, and I.A.N. Nimmo-smith, "The structure of normal human attention: The Test of Everyday Attention," pp. 525534,1996

13 A. Molotanovs, "Sacensību darbības optimizēšana handbola vārtsargiem (uz HK LSPA komandas piemēra)," Latvijas Sporta pedagoǵijas akadēmija, 2009

14 A. Hurtig-Wennlöf, M. Hagströmer, and L.A. Olsson, "The International Physical Activity Questionnaire modified for the elderly: aspects of validity and feasibility," Public Health Nutr., 13, no. 11, pp. 1847-1854, Nov. 2010

15 FINBALT, "Heath Behaviour among Latvian Adult Population, 2008," 2008

16 E. Iuliano, A. di Cagno, G. Aquino, G. Fiorilli, P. Mignogna, G. Calcagno, and A. Di Costanzo, "Effects of different types of physical activity on the cognitive functions and attention in older people: A randomized controlled study," Exp. Gerontol., 70, pp. 105-110, 2015

17 K.H. Karlsgodt, "Working Memory," in Brain Mapping: An Encyclopedic Reference, 3, Elsevier Inc., 2015, pp. 319-326

18 V.B. Ignjatović, S. Kalabić, S. Batić, and M. Žikić, "Improvement of Cognitive Efficiency Through Cognitive Training in Healthy Subjects," Acta Clin. Croat., 54, no. 2, pp. 169-78, Jun. 2015 
